\title{
Producción de semillas de quebracho blanco (Aspidosperma quebracho-blanco) en un sector del Chaco semiárido y su relación con el tamaño del fruto
}

\author{
Seed production in relation to fruit size of quebracho blanco (Aspidosperma quebracho-blanco) \\ in the semiarid Chaco forest
}

\author{
Andrés Tálamo a*, Silvana Cardozo ${ }^{\mathrm{b}}$, Federico Mohr ${ }^{\mathrm{c}}$ \\ *Autor de correspondencia: ${ }^{a}$ Universidad Nacional de Salta (UNSa), Consejo Nacional de Investigaciones Científicas y Técnicas \\ (CONICET) - Instituto de Bio y Geociencias del Noroeste Argentino (IBIGEO), Avenida Bolivia 5150, \\ Salta, Argentina, tel.: 54-387-154 448066, atalamo@unsa.edu.ar

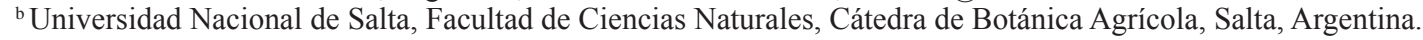 \\ c Universidad Nacional de Salta, Facultad de Ciencias Naturales, Salta, Argentina.
}

\begin{abstract}
SUMMARY
Effective management and conservation of a biological population requires knowledge of the different phases of its life cycle. We present data on seed production of quebracho blanco (white quebracho), Aspídosperma quebracho-blanco, a key tree species in the Chaco ecosystem of South America. In the southwestern sector of Copo National Park, Argentina, we recorded numbers of viable (with embryo) and non-viable (without embryo) seeds per fruit. The average fruit held 23 viable and 2 non-viable seeds (medians). Using a simple regression model we determined the relation between number of viable seeds and fruit size (width and length, respectively): $b_{\text {width }}=4.9, \mathrm{R}^{2}=0.18 ; b_{\text {length }}=0.35, \mathrm{R}^{2}=0.33$. The low goodness-of-fit values indicate that size is not a reliable tool for accurate predictions of the number of viable seeds per fruit. Nevertheless, given the positive slopes of the two regressions, we recommend that field workers collecting quebracho blanco seeds for regeneration select large fruits when possible. For further knowledge of this life history phase (seed production) and for estimations of the regeneration potential of quebracho blanco, we suggest that future studies focus on the variation in number of fruits produced per tree.
\end{abstract}

Key words: capsule, semiarid forest, viable seeds, white quebracho

\section{RESUMEN}

Para manejar y conservar una población biológica es necesario conocer las etapas de su ciclo de vida. El quebracho blanco es una especie clave en el ecosistema chaqueño y es necesario emprender estudios relacionados con su producción de semillas. Se estudió el número de semillas fértiles (con embrión) y vanas por fruto (sin embrión) y la relación entre el número de semillas fértiles con el tamaño (largo y ancho en cm) del fruto del quebracho blanco en el sector sur-oeste del Parque Nacional Copo, Argentina. Se encontró un promedio de 23 semillas fértiles y dos semillas vanas por fruto. El ancho y el largo del fruto estuvieron positivamente asociados con el número de semillas fértiles por fruto $\left(\mathrm{b}_{\text {ancho }}=4,9, \mathrm{R}^{2}=0,18 ; \mathrm{b}_{\text {largo }}=0,35, \mathrm{R}^{2}=0,33\right)$. Los modelos no serían una buena herramienta para predecir el número de semillas fértiles por fruto debido a sus bajos valores de bondad de ajuste. Sin embargo, al momento de seleccionar frutos con el objetivo de recolectar semillas, se recomienda seleccionar frutos de mayor tamaño. Se sugiere continuar el estudio mediante una estimación del número de frutos promedio por árbol para terminar de comprender esta etapa del ciclo de vida y estimar el potencial de regeneración del quebracho blanco.

Palabras clave: bosque chaqueño, cápsula, semillas viables, quebracho blanco.

\section{INTRODUCCIÓN}

Aspidosperma quebracho-blanco Schlecht, Apocinaceae (quebracho blanco), es una de las especies clave del bosque chaqueño semiárido, dado que junto con Schinopsis lorentzii (Griseb.) Engl, Anacardiaceae (quebracho colorado), son las especies de árboles que le confieren a este ambiente el estrato arbóreo alto y el nombre con el que se conoce a esta unidad fisonómica: "quebrachal de dos quebrachos" (Morello y Adámoli 1974). El quebracho blanco es además la especie que domina en densidad y área ba- sal en muchos sectores del Chaco semiárido, dejando en segundo lugar al quebracho colorado (Tálamo y Caziani 2003, Tálamo 2006).

Conocer los aspectos del ciclo de vida de una especie es importante para manejar y conservar sus poblaciones. A pesar de esto y de la importancia del quebracho blanco en el ambiente chaqueño, la información sobre aspectos reproductivos es escasa e incompleta. Se han realizado estudios sobre la estructura floral y los mecanismos de polinización (Lin y Bernardello 1999), sobre la calidad de las semillas en condiciones de laboratorio (Sandoval 
2006, Alzugaray et al. 2007), sobre la germinación natural y su dependencia de procesos facilitativos (Barchuk y Díaz 2000, Barchuk et al. 2005), y sobre la capacidad de rebrote y las estrategias de persistencia de la especie en su ambiente natural (Barchuk et al. 1999, Barchuk et al. 2006). Sin embargo, existe un vacío de información entre la polinización y el establecimiento de las plántulas, particularmente en relación con la producción de frutos por árbol y la producción de semillas por fruto. Una característica importante en la estimación del potencial reproductivo de una especie es el número y la proporción de semillas vanas o vacías (sin embrión). Entre las especies que presentan esta característica se encuentra el quebracho blanco y se ha propuesto como posible explicación la ocurrencia de excesivas precipitaciones y altas temperaturas durante la floración y formación de los frutos (Alzugaray et al. 2007). Sería muy valioso completar esta información sobre la historia natural del quebracho blanco para ampliar el conocimiento y la comprensión de una etapa del ciclo de vida de esta especie que permitirá estimar su potencial neto de regeneración (i.e. número de semillas fértiles por unidad de superficie) para un sector determinado.

Por otro lado, conocer la relación entre el tamaño del fruto y el número de semillas por fruto sería de gran utilidad para (1) disponer de una herramienta de predicción que permita estimar la producción de semillas por fruto simplemente conociendo su tamaño (midiendo solamente el largo o el ancho), mediante un método no destructivo (Pece et al. 2008) y (2) decidir sobre el tamaño de los frutos a cosechar con fines de propagación (Rocas et al. 2007, Morales-Velázquez 2010). La bibliografía informa una relación positiva entre el número de semillas y el tamaño del fruto (Devadas et al. 1999, Nieto de Pascual 2003, Chao 2005, Mápula-Larreta et al. 2007, Sivacioglu y Ayan 2008, Pece et al. 2008), ya que el número de semillas jugaría un rol importante en la demanda de hormonas que la planta destinaría al crecimiento del fruto (Harris 1912, Devadas et al. 1999).

Los objetivos de este trabajo son: 1) estimar el número promedio de semillas fértiles y de semillas vanas por fruto y 2) estudiar la variación del número de semillas fértiles por fruto en función del tamaño del mismo en quebracho blanco (A. quebracho-blanco) en un sector de Chaco semiárido argentino.

\section{MÉTODOS}

El estudio se realizó en el sector sur-oeste del Parque Nacional Copo (Santiago del Estero, Argentina), bosque chaqueño semiárido que corresponde a la subregión de los Antiguos Cauces del Río Juramento-Salado (Morello y Adámoli 1974). La vegetación dominante en superficie es un bosque semidecíduo de quebracho blanco, quebracho colorado y mistol (Zyziphus mistol Griseb., Rhamnaceae), interrumpido por pastizales asociados a paleocauces. El clima es marcadamente estacional, con $80 \%$ de las preci- pitaciones anuales $(700 \mathrm{~mm})$ concentradas entre octubre y marzo (primavera-verano) (Bianchi y Yáñez 1992). Para una descripción más completa de la vegetación leñosa consultar Tálamo y Caziani (2003) y Tálamo (2006).

Dentro del sector estudiado de aproximadamente 2.500 hectáreas de bosque continuo (sin posibilidades de diferenciar poblaciones o rodales), se seleccionaron 230 frutos provenientes de 11 individuos adultos de quebracho blanco (distanciados por no menos de 500 metros entre sí) que presentaron una oferta abundante de frutos. Los frutos, cápsulas oblongas de forma elíptica, fueron cosechados antes de su dehiscencia natural (para evitar la pérdida de semillas dispersadas por el viento) y luego almacenados en cajones hasta la apertura de los mismos por la pérdida de humedad. Para cada fruto se registró: a) el largo (longitud entre la base y el ápice, en $\mathrm{cm}$ ) y el ancho máximo (cm) mediante un calibre (precisión $0,05 \mathrm{~mm}$ ) y se contó el número de semillas fértiles (con embrión) y el número de semillas vanas (sin embrión), de clara diferenciación visual entre sí con sólo observar la semilla a trasluz.

Análisis estadísticos. Para caracterizar la tendencia central del número de semillas fértiles y del número de semillas vanas por fruto, se calculó la mediana en vez de la media aritmética, ya que el conteo de semillas vanas por fruto mostró una distribución muy asimétrica (Quinn y Keough 2002). Asimismo la variabilidad de estos valores a través de los frutos se cuantificó con el rango intercuartil (diferencia entre el cuartil 3 y el cuartil 1). Además se utilizó la técnica de bootstrap para calcular los límites del intervalo de confianza (95\%) para la mediana (Manly 1997). La relación entre el número de semillas fértiles (variable dependiente) con el largo y el ancho del fruto (variables independientes) se evaluó con un análisis de regresión lineal simple para cada variable independiente. La variable dependiente "número de semillas fértiles" presentó una distribución normal (coeficiente de asimetría de Pearson = $0,098)$ y sus residuales no mostraron relación con los valores predichos por el modelo (Quinn y Keough 2002). No se utilizó un modelo de regresión múltiple porque ambas variables independientes estuvieron positivamente correlacionadas $(\mathrm{r}=0,74 ; P<0,001)$, violándose uno de los supuestos de dicha técnica (Quinn y Keough 2002). Los gráficos y los análisis estadísticos fueron realizados con el programa libre R (R Development Core Team 2010).

\section{RESULTADOS}

Los frutos de quebracho blanco cosechados presentaron un ancho promedio de $5,2 \mathrm{~cm}$ y un largo promedio de $8,1 \mathrm{~cm}$, y ambas variables estuvieron positivamente correlacionadas entre sí (figura 1A). Se registró un promedio de 23 semillas fértiles y dos semillas vanas por fruto (figura 1B) y un 5,5\% de semillas vanas por fruto (rango intercuartil $=29,1 \%$ ). Al extrapolar los resultados a la población de frutos del sector estudiado, se puede inferir con 

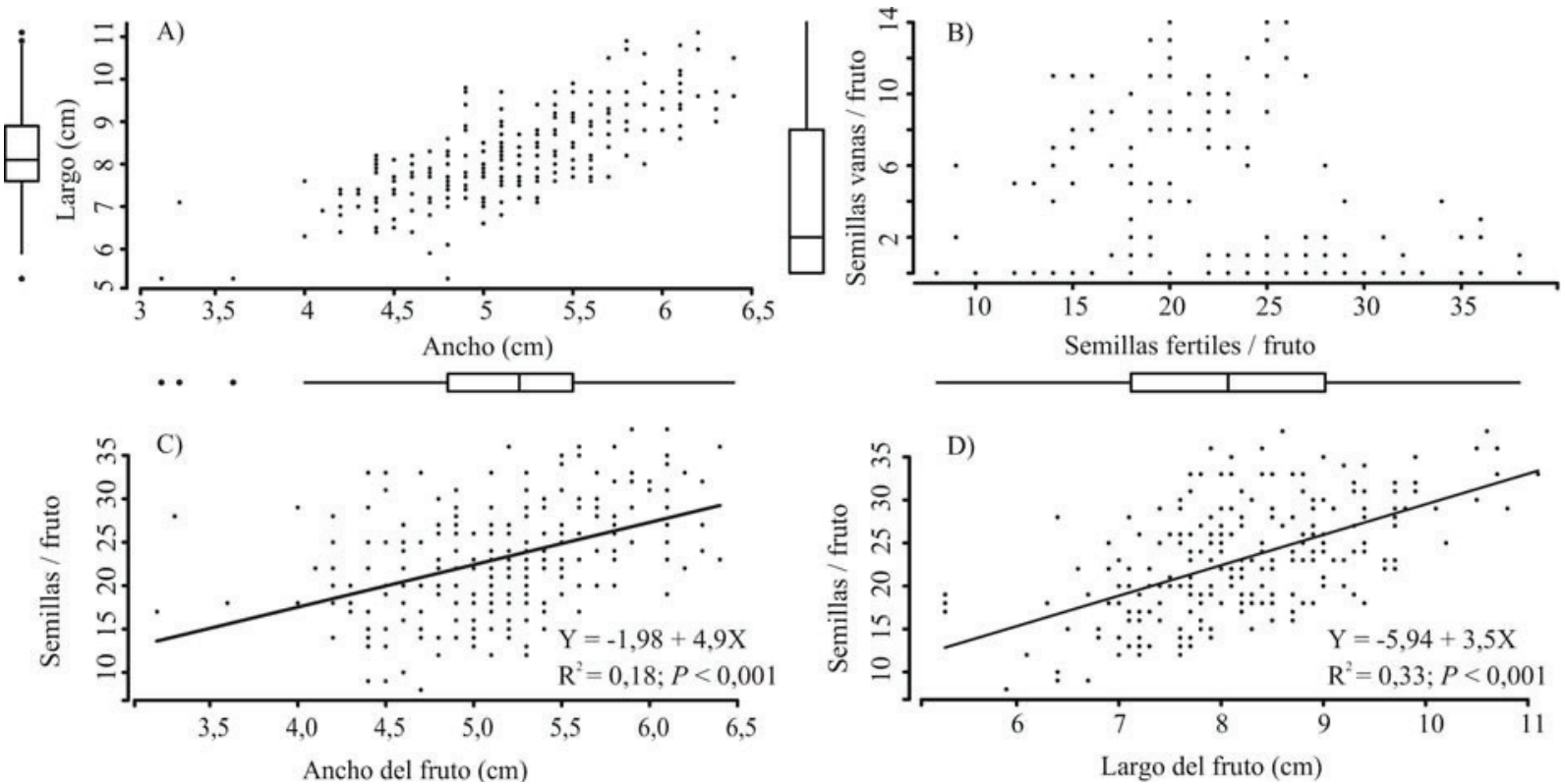

Figura 1. A) Relación entre el largo y el ancho de frutos de quebracho blanco, B) relación entre el número de semillas vanas y fértiles por fruto, C) relación lineal simple del número de semillas fértiles por fruto con el ancho del fruto y D) relación lineal simple del número de semillas fértiles por fruto con el largo del fruto. Muestra de 230 frutos recolectados en un sector del Parque Nacional Copo, Santiago del Estero, Argentina. Se presentan además los gráficos de caja de cada variable.

A) Relationship between fruit length and fruit width, B) relationship between the number of viable and non-viable seeds per fruit, C) simple linear regression between the number of viable seeds per fruit and fruit width and D) simple linear regression between the number of viable seeds per fruit and fruit length. Sample size: 230 quebracho blanco fruits (Aspidosperma quebracho-blanco) collected in the southwestern sector of Copo National Park, Santiago de Estero, Argentina.

un $95 \%$ de confianza, que el ancho promedio de los frutos estaría comprendido entre 5,13 y $5,27 \mathrm{~cm}$, el largo promedio entre 7,96 y $8,24 \mathrm{~cm}$, el número de semillas fértiles por fruto entre 22 y 24 semillas, el número de semillas vanas por fruto entre 0,4 y 4 semillas y el porcentaje de semillas vanas por fruto entre 3 y $7,6 \%$.

El número de semillas fértiles estuvo relacionado positiva y significativamente con el ancho $(\mathrm{t}=7,3 ; P<0,001)$ $\mathrm{y}$ con el largo del fruto $(\mathrm{t}=10,7 ; P<0,001)$ (figura $1 \mathrm{C}$ y 1D). El estimador puntual de la pendiente cuando se utiliza el ancho del fruto como variable independiente fue de 4,9 semillas $\mathrm{cm}^{-1}$ y se puede inferir con un $95 \%$ de confianza que la pendiente poblacional estaría comprendida entre 3,6 y 6,2 semillas $\mathrm{cm}^{-1}$. Por otro lado, al utilizar el largo del fruto como variable independiente la estimación puntual de la pendiente fue de 3,5 semillas $\mathrm{cm}^{-1}$, y se infiere con un $95 \%$ de confianza que la pendiente poblacional estaría comprendida entre 2,9 y 4,2 semillas $\mathrm{cm}^{-1}$.

\section{DISCUSIÓN}

Las dimensiones de los frutos medidos coinciden en gran medida con lo informado por Digilio y Legname (1966) para esta especie: 7 a $11 \mathrm{~cm}$ de longitud y 4 a $6 \mathrm{~cm}$ de ancho. En el Chaco Boliviano, los frutos medidos por Sandoval (2006) fueron de menor tamaño (largo de $5-9 \mathrm{~cm}$ y ancho de $4-6 \mathrm{~cm}$ ), diferencias que posiblemente estén explicadas por cuestiones climáticas. Se menciona para quebracho blanco una considerable variabilidad genética intraespecífica, por lo que las diferencias en ciertas estructuras podrían considerarse como plasticidad fenotípica (Barchuk et al. 2006). En cuanto al porcentaje del número de semillas vanas por fruto, los valores encontrados en la muestra analizada del bosque chaqueño semiárido (5\%) coinciden con lo informado para otros estudios realizados en el Chaco húmedo santafesino, en donde Alzugaray et al. (2007) encontraron un $4 \%$ de semillas sin embrión.

Existe muy poca información sobre la relación entre el tamaño del fruto y el número de semillas por fruto en plantas del bosque chaqueño. En este ambiente, Pece et al. (2008) reportaron una relación positiva y significativa para el algarrobo blanco (Prosopis alba Griseb), aunque se desconoce el grado de ajuste del modelo estimado para poder evaluar su utilidad como herramienta de predicción. En cuanto a las pendientes estimadas en este trabajo $\left(\mathrm{b}_{\text {ancho }}=4,9 ; \mathrm{b}_{\text {largo }}=3,5\right)$, las mismas indican que por cada centímetro de aumento en el ancho del fruto, el número de semillas fértiles aumenta 4,9 unidades, mientras que por cada centímetro de aumento en el largo del fruto, el número de semillas se incrementa en 3,5 unidades. Sin embargo, el grado de ajuste de ambos modelos no fue muy elevado $\left(\mathrm{R}_{\text {ancho }}^{2}=18 \%, \mathrm{R}_{\text {largo }}^{2}=33 \%\right)$ por lo que no sería de gran utilidad usar dichos modelos para predecir el número de semillas fértiles por fruto en función de su tamaño. Por 
lo tanto, para un determinado ancho o una determinada longitud de fruto, existe un rango muy amplio de posibles valores predichos del número de semillas fértiles por fruto. Aunque los modelos estimados no constituyen una herramienta con alto valor de predicción, la relación positiva encontrada permite recomendar la cosecha de frutos de mayor tamaño para obtener un mayor número de semillas fértiles. De manera similar, también se ha recomendado recolectar frutos de mayor tamaño para obtener un mayor rendimiento de semillas desarrolladas para el caso de otras especie de interés forestal en bosques de México (Rocas et al. 2007, Morales-Velázquez et al. 2010).

Este trabajo constituye el primer estudio en cuantificar el número de semillas por fruto y su relación con el tamaño de los frutos en quebracho blanco contribuyendo a completar la descripción de la morfometría del fruto de una población de individuos adultos del sur-oeste del Parque Nacional Copo, Santiago del Estero, Argentina.

Se recomienda continuar estudiando esta etapa del ciclo de vida del quebracho blanco, indagando sobre un aspecto importante pendiente: la producción de frutos por árbol de la zona de estudio. Conociendo (1) el número de frutos por árbol y su variabilidad entre árboles, (2) la abundancia individuos de quebracho blanco por unidad de superficie (Tálamo 2006) y (3) el número de semillas fértiles por fruto estimado en este trabajo, podríamos conocer la producción de semillas fértiles por unidad de superficie, información clave para comprender el potencial de regeneración natural del quebracho blanco en el sector sur oeste del Parque Nacional Copo, Santiago del Estero, Argentina.

\section{AGRADECIMIENTOS}

Agradecemos a la Administración de Parques Nacionales por la autorización y el apoyo brindado para el desarrollo del presente trabajo. Además estamos especialmente agradecidos con Ricardo Pérez por el apoyo brindado para la cosecha de los frutos de quebracho blanco y con M. Núñez-Regueiro por su constante motivación. El apoyo financiero provino de un subsidio de The Rufford Small Grants Foundation otorgado al primer autor. Dos revisores anónimos mejoraron sustancialmente una versión original del presente manuscrito.

\section{REFERENCIAS}

Alzugaray C, NJ Carnevale, AR Salinas. 2007. Evaluación de la calidad de semillas de Aspidosperma quebracho-blanco Schlecht, y Schinopsis balansae Engl. mediante la prueba de rayos X. Revista de Investigación, Facultad de Ciencias Agrarias 11: 51-59.

Alzugaray C, NJ Carnevale, AR Salinas, R Pioli. 2006. Calidad de semillas de Aspidosperma quebracho-blanco Schlecht. Quebracho 13: 26-35.

Alzugaray C, NJ Carnevale, AR Salinas, R Pioli. 2007. Factores bióticos y abióticos que afectan la calidad de las semillas de Schinopsis balansae Engl. y Aspidosperma quebracho- blanco Schltdl. Revista Iberoamericana de Micología 24: 142-147.

Barchuk AH, MP Díaz. 1999. Regeneration and structure of Aspidosperma quebracho-blanco Schl. in the Arid Chaco (Córdoba, Argentina). Forest Ecology and Management 118: 31-36.

BarchukAH, MPDíaz. 2000. Vigor de crecimiento y supervivencia de plantaciones de Aspidosperma quebracho-blanco y de Prosopis chilensis en el Chaco árido. Quebracho 8: 17-29.

Barchuk AH, A Valiente-Banuet, MP Díaz. 2005. Effect of shrubs and seasonal variability of rainfall on the establishment of Aspidosperma quebracho-blanco in two edaphically contrasting environments. Austral Ecology 30: 695-705.

Barchuk AH, MR Iglesias, C Oviedo. 2006. Rebrote basal de Aspidosperma quebracho-blanco en estado de plántula: mecanismo de persistencia en el Chaco Árido. Ecología Austral 16: 197-205.

Bianchi AR, CE Yáñez. 1992. Las Precipitaciones en el Noroeste Argentino. Segunda Edición. Salta, Argentina. Instituto Nacional de Tecnología Agropecuaria (INTA). 383 p.

Chao CCT. 2005. Pollination study of mandarins and the effect on seediness and fruit size: Implications for seedless mandarin production. HortScience 40: 362-365.

Devadas VS, KJ Kuriakose, TG Rani, TR Gopalakrishnan, SR Fair. 1999. Influence of fruit size on seed content and quality in pumpkin (Cucúrbita moschata Poir). Seed Research 27(1): 71-73.

Digilio APL, PR Legname. 1966. Los árboles indígenas de la provincia de Tucumán. Opera Lilloana 15: 54.

Harris JA. 1912. The influence of the seed upon the size of the fruit in staphylea. IIIV. On the nature of the correlation between the number of seeds and pod length. Botanical Gazette 53: 396-414.

Lin S, G Bernardello. 1999. Flower structure and reproductive biology in Aspidosperma quebracho-blanco (Apocynaceae), a tree pollinated by deceit. International Journal of Plant Sciene 160(5): 869-878.

Manly BFJ. 1997. Randomization, bootstrap and Monte Carlo methods in biology. London, UK. Chapman \& Hall. Second edition. 399 p.

Mápula-Larreta M, J López-Upton, JJ Vargas-Hernández, A Hernández-Livera. 2007. Reproductive indicators in natural populations of Douglas-fir in Mexico. Biodiversity and Conservation 16: 727-742.

Morales-Velázquez MG, CA Ramírez-Nandujano, P DelgadoValerio, J López-Upton. 2010. Indicadores reproductivos de Pinus leiophylla Schltdl et. Chan en la cuenca del río Angulo, Michocán. Revista Mexicana de Ciencias Forestales 1(2): 31-38.

Morello J, J Adámoli. 1974. Las grandes unidades de vegetación y ambiente de la Provincia del Chaco. Bs. As., Argentina. INTA Serie Fitogeográfica 13. 122 p.

Nieto de Pascual P, CMA Musalem, J Ortega Alcalá. 2003. Estudio de algunas características de conos y semillas de Abies religiosa (HBK) Schl. et Cham. Agrociencia 37: 521531.

Pece M, M Juárez de Galíndez, M Acosta, CG de Benítez, S Saavedra, C Bruno. 2008. Relación entre la longitud de la vaina y el número de semillas por vaina en Algarrobo blanco. Quebracho 15: 59-63.

Quinn G, M Keough. 2002. Experimental Design and Data 
Analysis for Biologists. New York, USA. Cambridge University Press. 537 p.

R Development Core Team. 2010. R: A language and environment for statistical computing. R Foundation for Statistical Computing, Vienna, Austria. ISBN 3-900051-07-0, URL http://www.R-project.org

Rocas AN, EO Ramírez-García, A Aparicio-Rentería. 2007. Correlación entre características de frutos de Swietenia macrophylla King con su contenido de semillas desarrolladas. Foresta Veracruzana 9(1): 49-53.

Sandoval E. 2006. Ensayo de longevidad de semillas de quebracho blanco. Documento Científico Proyecto FOMABO n ${ }^{\circ} 2$. Santa Cruz, Bolivia. Proyecto FOMABO. 14 p.
Sivacioglu A, S Ayan. 2008. Evaluation of seed production of Scots pine (Pinus sylvestris L.) clonal seed orchard with cone analysis method. African Journal of Biotechnology 7(24): 4393-4399.

Tálamo A, SM Caziani. 2003. Variation in woody vegetation among sites with different disturbance histories in the Argentine Chaco. Forest Ecology and Management 184: 79-92.

Tálamo A. 2006. Biodiversidad de plantas leñosas y disturbios humanos en el bosque chaqueño semiárido: efectos del aprovechamiento forestal. Tesis de Doctorado. Buenos Aires, Argentina. Facultad de Ciencias Exactas y Naturales, Universidad de Buenos Aires. 110 p.

Recibido: 04.07.11

Aceptado: 24.10.11 
